\title{
Sensitivity and Spatial Resolution of Transvenous Leads in Implantable Cardioverter Defibrillator
}

\author{
Jesús Requena-Carrión*, Member, IEEE, Juho Väisänen, Felipe Alonso-Atienza, Member, IEEE, \\ Arcadi García-Alberola, Francisco Javier Ramos-López, Member, IEEE, \\ and José Luis Rojo-Álvarez, Member, IEEE
}

\begin{abstract}
It has been previously documented that the main features and sensing performance of electrograms (EGMs) recorded in implantable cardioverter defibrillators (ICDs) depend on lead configuration. Although this dependence has been ascribed to differences in lead sensitivity and spatial resolution, the quantification of these two properties on ICD has not yet been attempted. In this paper, an operative framework to study the spatial resolution of ICD transvenous leads is presented. We propose to quantify the spatial resolution of ICD transvenous leads based on a new characterization called lead resolution volume (ResV). We analyzed the sensitivity distribution and the ResV of two unipolar (tip-can and coil-can) and two bipolar (true or tip-ring and integrated or tipcoil) ICD transvenous lead configurations. A detailed 3-D model of the human thorax based on the visible human man dataset was used to compute the lead sensitivity and computer simulations of simple cardiac dynamics were used to quantify the ResV. Differences in the sensitivity distribution throughout the ventricular myocardium (VM) were observed for each lead configuration. In our computer model of the human thorax, the ResV was found to comprise $7 \%, 35 \%, 45 \%$, and $70 \%$ of the VM for true bipolar, integrated bipolar, tip-can unipolar, and coil-can unipolar ICD leads, respectively. Furthermore, our analysis shows that the spatial resolution depends on both lead sensitivity and cardiac dynamics, and therefore, it can vary for different heart rhythms.
\end{abstract}

Index Terms-Bipolar lead, implantable cardioverter defibrillator (ICD), lead field, resolution volume, sensitivity, spatial resolution, transvenous leads, unipolar lead.

\section{INTRODUCTION}

$\mathbf{T}$ HE implantable cardioverter defibrillator (ICD) is a lifesupporting device that continuously monitors cardiac rhythm and automatically delivers an electrical therapy after

Manuscript received November 26, 2008; revised February 19, 2009 and May 6 , 2009. First published July 28, 2009; current version published November 20, 2009. This work was supported in part by the Sociedad Española de Cardiología under research grants and in part by the Spanish Ministry of Science and Innovation under Research Project TEC2007-68096-CO2/TCM. Asterisk indicates corresponding author.

${ }^{*} \mathrm{~J}$. Requena-Carrión is with the Department of Signal Theory and Communications, Rey Juan Carlos University, Madrid 28943, Spain (e-mail: jesus. requena@urjc.es).

J. Väisänen is with the Department of Biomedical Engineering, Tampere University of Technology (TUT), Tampere 33101, Finland (e-mail: juho. vaisanen@tut.fi).

F. Alonso-Atienza, F. J. Ramos-López, and J. L. Rojo-Álvarez are with Department of Signal Theory and Communications, Rey Juan Carlos University, Madrid 28943, Spain (e-mail: felipe.alonso@urjc.es; javier.ramos@urjc.es; joseluis.rojo@urjc.es).

A. García-Alberola is with the Arrhythmia Unit, Hospital Universitario Virgen de la Arrixaca, Universidad de Murcia, Murcia 30120, Spain (e-mail: arcadi@secardiologia.es).

Color versions of one or more of the figures in this paper are available online at http://ieeexplore.ieee.org.

Digital Object Identifier 10.1109/TBME.2009.2027425 detecting a lethal arrhythmia. Nowadays, most ICD use transvenous leads that are inserted into the cardiac chambers to sense electrogram (EGM) signals induced by the electrical activity of the heart [1]. According to previous studies, ICD lead configurations can affect fundamental EGM features, such as wave duration and amplitude, and spectral content [2]-[5]. It has also been shown that lead configuration can affect ICD sensing performance. It was reported in [6] that pacing stimulus artifacts, often associated to ICD undersensing, were greater in integrated than in true bipolar leads. Other studies have reported differences in ventricular fibrillation detection and redetection times when comparing ICD integrated and true bipolar leads [7]-[10]. Although these discrepancies are often ascribed to differences in lead sensitivity and spatial resolution in the ventricular myocardium (VM), to date no quantitative description of both properties has been attempted on ICD. Estimating the size and distribution of the spatial resolution of ICD leads in the VM would improve current ICD sensing performance and widen its range of functionality, especially when the underlying cardiac pathology is caused by physiological abnormalities locally in the VM. In Brugada patients, for instance, it has been observed that some arrhythmic episodes are preceded by anomalous depolarizations in the right ventricular outflow tract [11]. Therefore, the early detection of these arrhythmic episodes from EGM analysis, would only be possible if it were known that the spatial resolution of the lead covers the right ventricular outflow tract. In a similar way, implantable technology has been recently proposed for detecting and monitoring myocardial ischemia [12]-[14]. In this scenario, an estimation of the spatial resolution would help in determining what ischemic regions within the myocardium could be sensed by the leads.

In this paper, we present an operative framework to quantify the spatial resolution of ICD transvenous leads based on a new characterization called the lead resolution volume (ResV). The Res V is defined as the smallest myocardial region that induces an EGM component at the lead that differs from the total EGM by less than a specified fraction of its mean power. The aim of our study is to compare the sensing capabilities of different lead configurations to ventricular physiological events and consequently, we restrict our analysis to the VM. We analyze both the sensitivity distribution and the ResV of two unipolar and two bipolar ICD transvenous leads. First, we compute the sensitivity distribution of each ICD lead on a detailed 3-D model of the human thorax. Then, we identify the isosensitivity surfaces within the VM, and finally, we use computer simulations to estimate the ResV in the VM. 


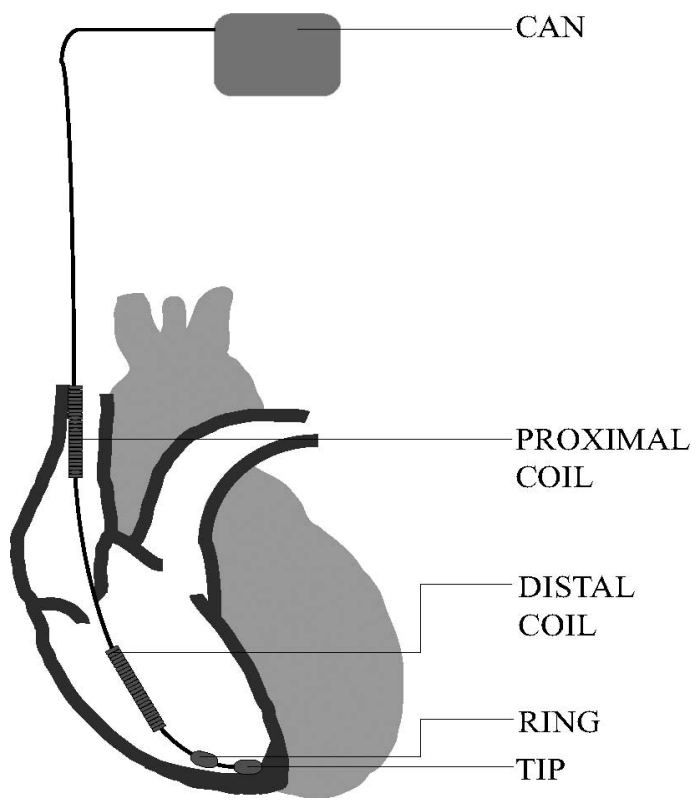

Fig. 1. ICD transvenous lead systems consist of a set of electrodes that are guided through a vein into the heart chambers (tip, ring, or distal coil) in combination with the casing of the implant (active can) and, when available, with the proximal coil.

The paper is organized as follows. Section II provides a background on ICD lead technology, sensitivity, and spatial resolution. In Section III, the notion of ResV is presented. Section IV describes the methods used to estimate the sensitivity and ResV of ICD leads. In Section V, the results of our study are presented, and finally, in Section VI, we discuss the scope and limitations of our approach and future extensions.

\section{BACKGROUND}

\section{A. ICD Transvenous Leads}

The ICD performs two basic tasks, namely tachyarrhythmia detection and tachyarrhythmia termination. The former is mostly based on the analysis of heart rhythm from intracardiac EGM sensed at the leads, and the latter consists of restoring a normal heart rhythm by delivering an electrical therapy such as cardiac defibrillation, from the leads. Nowadays, most ICD use transvenous leads, which consist of a set of electrodes that are guided through a vein into the heart chambers, in combination with the casing of the ICD [1] (see Fig. 1). Depending on the manufacturer and the model, some of the following electrodes are used for the basic functionality of ventricular tachyarrhythmia detection and termination [15], [16]:

1) Tip: It is the most distal of the electrodes, and has a small surface and is located in the apex of the right ventricle (RV).

2) Ring: It is located a few millimeters away from the tip electrode, and has a small surface.

3) Distal coil: It is an elongated electrode located in the RV, close to the tip, and runs parallel to the septum.

4) Proximal coil: It is similar to the distal coil, but is located in the superior cava vein.
5) Active can: It is the casing of the implant, and is located in a left pectoral pocket and has a large surface.

By combining two or more electrodes, an electrical path is created that can be used for both EGM sensing in tachyarrhythmia detection and delivering therapy for tachyarrhythmia termination. Depending on the electrodes involved in EGM sensing, the following lead configurations are distinguished [15], [16]: unipolar configurations, consisting of one distal electrode (either the tip electrode or the distal coil) and the active can which is shortcut when available, with the proximal coil; true bipolar configurations, formed by the tip electrode and the ring electrode; and integrated bipolar configurations, which use the tip and the distal coil. ICD automatic detection algorithms based on heart rhythm criteria usually rely on the analysis of bipolar EGM to determine whether a life-threatening arrhythmia exists.

\section{B. Lead Sensitivity and Spatial Resolution}

The lead sensitivity distribution, or lead field, describes the ability of a lead to measure bioelectric sources distributed throughout a volume conductor. Let $\mathbf{J}(v, t)$ be a distribution of time-varying dipole sources on a volume conductor $V$, where $v$ is a point within the volume conductor $V$ and $t$ denotes time. According to lead field theory [17], signal $z(t)$ recorded by a lead that is characterized by a sensitivity distribution $\mathbf{L}(v)$ on the volume conductor $V$, can be expressed as follows:

$$
z(t)=\int_{V} \mathbf{L}(v) \cdot \mathbf{J}(v, t) d v
$$

This equation states that the signal recorded by a lead is the linear combination of the dipole sources $\mathbf{J}(v, t)$ weighted by lead sensitivity distribution $\mathbf{L}(v)$.

The analysis of the sensitivity distribution has been useful in electrophysiology to investigate qualitatively the spatial resolution of leads, i.e., the region of the bioelectric source that contributes the most to the recorded signal. In an early work by Rush and Driscoll in 1969, the sensitivity of electroencephalographic (EEG) leads was studied [18]. Later, Arzbaecher investigated the sensitivity within the heart of an unipolar precordial electrode and esophageal unipolar and bipolar electrodes [19]. Lead sensitivity has also made quantifying lead spatial resolution possible. The half sensitivity volume was proposed in [20] to compare the spatial resolution of EEG and magnetoencephalographic systems. The region of interest sensitivity ratio was defined in [21] as the ratio between the average sensitivities of two regions, in order to quantify the specificity of EEG measurements on the region of interest sources. Finally, the concept of Res $V$ was proposed in [22] for estimating the spatial resolution of surface electrocardiographic (ECG) lead systems.

From the analysis of the magnitude of the sensitivity distribution of bipolar and unipolar ICD leads [5], it is widely accepted that the former sense electrical events from smaller bioelectric regions than the latter. Nevertheless, despite of the importance of the notions of sensitivity and spatial resolution, little attention has been paid to their quantification in ICD transvenous leads. 


\section{QUANTIFICATION OF ICD SENSITIVITY AND RESV}

In this section, we present our approach to the study of the sensitivity distribution and the spatial resolution of ICD transvenous lead. The lead sensitivity distribution is analyzed using lead isosensitivity surfaces throughout the VM, and the spatial resolution is quantified by estimating the ResV in the VM. The mathematical formulation of the Res $\mathrm{V}$, previously proposed by our group to investigate the sensitivity properties of surface ECG [22], and a numerical method for its estimation based on computer simulations are next described.

\section{A. Isosensitivity Surfaces}

In order to analyze ICD lead's sensitivity distribution, a family of isosensitivity surfaces throughout the VM can be obtained for every lead configuration under investigation. An isosensitivity surface $S_{r}$ is a collection of points $v$ in the volume conductor $V$ where the magnitude of the lead sensitivity remains constant. Mathematically, an isosurface is defined as

$$
S_{r}=\left\{v \in V,\|\mathbf{L}(v)\|=L_{r}\right\}
$$

where $L_{r}$ is a reference value for the sensitivity and $r$ is an index. A family of isosensitivity surfaces can be obtained for different values of $L_{r}$. Isosensitivity surfaces can help visualize the regions within the VM where the sensitivity distribution of ICD transvenous lead is highest and how it decreases with the distance to the electrodes. Nevertheless, the vectorial nature of the lead sensitivity distribution is not taken into consideration by using this description, since only its magnitude is depicted.

\section{B. Lead Resolution Volume}

The ResV is a numerical characterization of the spatial resolution that uses the discretized version of (1) for calculating the similarity between the EGM induced at the lead by the whole VM and the EGM component induced at the lead by smaller regions within the VM.

Let $V$ be a discretized model of the VM consisting of $N_{V}$ nodes and $V_{O}$ a region within the VM, $V_{O} \subseteq V$. From (1), if $V_{C}$ is the complementary region of $V_{O}$ with respect to $V$, i.e., if $V_{O}$ and $V_{C}$ are two disjoint regions such that $V=V_{O} \cup V_{C}$, then $\operatorname{EGM} z[k]$ can be decomposed as follows:

$$
\begin{aligned}
z[k] & =\sum_{v=1}^{N_{V}} \mathbf{L}[v] \cdot \mathbf{J}[v, k] \\
& =\sum_{v=1}^{N_{V_{O}}} \mathbf{L}[v] \cdot \mathbf{J}[v, k]+\sum_{v=1}^{N_{V_{C}}} \mathbf{L}[v] \cdot \mathbf{J}[v, k] \\
& =z_{O}[k]+z_{C}[k]
\end{aligned}
$$

where $k$ denotes the discretized time instant, $N_{V_{O}}$ and $N_{V_{C}}$ are the number of nodes in $V_{O}$ and $V_{C}$, so that $N_{V}=N_{V_{O}}+N_{V_{C}}$, and $z_{O}[k]$ and $z_{C}[k]$ are two components of the EGM induced, respectively, by myocardial regions $V_{O}$ and $V_{C}$ (see Fig. 2).

For every $V_{O}$, the similarity between EGM $z[k]$ and EGM component $z_{O}[k]$ is defined in terms of their mean square dif-
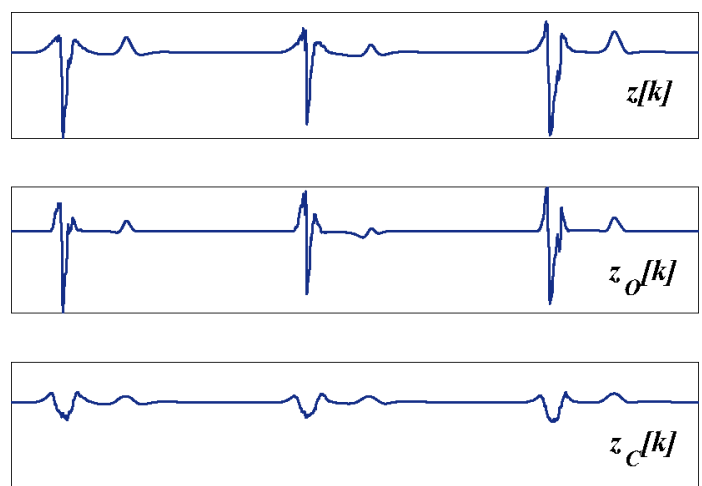

Fig. 2. Simulation of an EGM $z[k]$ induced by the whole VM, denoted by $V$, and two EGM components, $z_{O}[k]$ and $z_{C}[k]$, induced by two complementary myocardial regions, $V_{O}$ and $V_{C}$, respectively.

ference (MSD) as

$$
\begin{aligned}
\operatorname{MSD}\left\{V_{O}\right\} & =E\left[\left(z[k]-z_{O}[k]\right)^{2}\right] \\
& =E\left[\left(z_{C}[k]\right)^{2}\right]=P_{z_{C}}
\end{aligned}
$$

where $E[\cdot]$ denotes statistical mean and $P_{z_{C}}$ is the mean power of EGM component $z_{C}[k]$. In order to obtain a more convenient measure of similarity between EGM $z[k]$ and EGM component $z_{O}[k]$, we divide $\operatorname{MSD}\left\{V_{O}\right\}$ by the mean power of $z[k], P_{z}=$ $E\left[(z[k])^{2}\right]$, and define the normalized MSD between $z[k]$ and $z_{O}[k]$ as

$$
\operatorname{MSD}_{n}\left\{V_{O}\right\}=\frac{\operatorname{MSD}\left\{V_{O}\right\}}{P_{z}}=\frac{P_{z_{C}}}{P_{z}} .
$$

Therefore, $\operatorname{MSD}_{n}\left\{V_{O}\right\}$ can be seen as a measure of the error of approximating $z[k]$ to component $z_{O}[k]$, and alternatively, it can also be interpreted as a measure of the contribution of $V_{O}$ to $z[k]$. According to (5), the smaller $\operatorname{MSD}_{n}\left\{V_{O}\right\}$, the greater the contribution of $V_{O}$ to EGM $z[k]$. Evidently, if $V_{O}=V$, it follows that $\operatorname{MSD}_{n}\left\{V_{O}\right\}=0$, whereas if $V_{O}$ is infinitesimally small then $\operatorname{MSD}_{n}\left\{V_{O}\right\}$ approaches 1 .

In order to characterize the spatial resolution using the ResV, we define a threshold reference value $\alpha$ for the variable $\operatorname{MSD}_{n}\left\{V_{O}\right\}$ under which the contribution of $V_{O}$ can be considered to be significant. Note that there exist infinite regions $V_{O}$ that satisfy $\operatorname{MSD}_{n}\left\{V_{O}\right\} \leq \alpha$. For a region $V_{O}$ to be considered the ResV, we additionally impose that $V_{O}$ has the smallest size. Therefore, determining the ResV is equivalent to finding the smallest myocardial region that induces at the lead, an EGM component that taking the normalized MSD as a measure of discrepancy, differs from the total EGM by no more than a fraction $\alpha$ of its mean power.

\section{Estimation of ResV}

Since the statistical descriptions of EGM $z[k]$ and EGM component $z_{O}[k]$ are usually unknown, we estimate the ResV by means of computer simulations. By simulating the electrical activation of the VM we obtain a time-varying cardiac dipole distribution. Then, for every lead configuration we proceed as follows. First, a family of nested myocardial regions $\left\{V_{O}\right\}_{r}$ is 

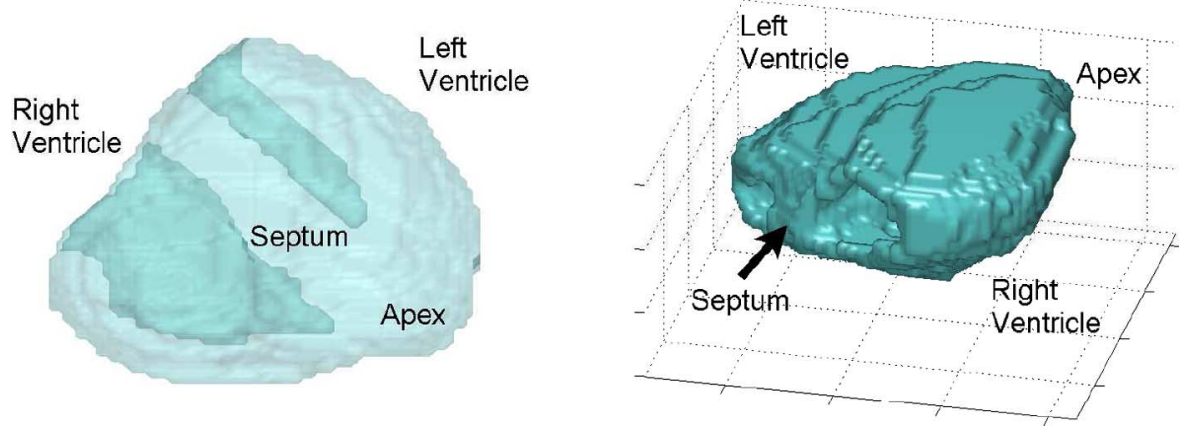

Fig. 3. Numerical model of the anatomy of the human VM based on the VHM dataset. This model serves as the anatomical substrate for the model of the cardiac electrical activity.

considered in which every region is bounded by an isosensitivity surface $S_{r}$ of progressively smaller magnitude, $L_{r}>L_{r+1}$. Then, EGM $z[k]$ and a family of EGM components induced by each region in $\left\{V_{O}\right\}_{r},\left\{z_{O}[k]\right\}_{r}$, are synthesized by using (3) and statistical averages are substituted by temporal averages in (5) in order to calculate for every region its contribution $\operatorname{MSD}_{n}\left\{V_{O}\right\}_{r}$. Finally, given a reference value $\alpha$ the $\operatorname{ResV}$ is estimated by determining the myocardial region of minimum size $V_{\text {min }} \in\left\{V_{O}\right\}_{r}$ that satisfies $\operatorname{MSD}_{n}\left\{V_{\min }\right\} \leq \alpha$.

\section{Bioelectric Model AND Simulations}

We used numerical methods to analyze the sensitivity distribution and the spatial resolution of four ICD transvenous leads. Since we were interested in comparing the sensing capabilities of each lead configuration to ventricular physiological events, we focused our analysis on the VM. First, we computed the sensitivity distribution in the VM using numerical models of both the human thorax and ICD transvenous leads. Second, we simulated the electrical activity of the VM using a cardiac activation computer model in order to synthesize EGM signals for finally estimating the ResV.

\section{A. Human Thorax}

A bioelectric model of the human thorax specifies the geometry and the conductive properties of the volume conductor where electric potentials are induced as a consequence of the activity of bioelectric sources within it. In this study, we used a realistic 3-D male thorax based on the VHM dataset [23], [24]. We used the VHM dataset since it has been widely used in bioelectric applications, and hence, it allows for fair comparisons between studies from different groups. The data consisted of 95 segmented slices of the human thorax that resulted in a cubic grid with resolution $1.67 \mathrm{~mm} \times 1.67 \mathrm{~mm} \times 4 \mathrm{~mm}$ in the heart and $1.67 \mathrm{~mm} \times 1.67 \mathrm{~mm} \times 8 \mathrm{~mm}$ elsewhere. Altogether, our model of the human thorax contained 2.7 million nodes. After segmentation, 20 different organ and tissue types were identified and assigned resistivity values found in the literature [25]. VM consisted of more than 30000 nodes, and it was used as the anatomical substrate for our cardiac activation model (see Fig. 3).

\section{B. Transvenous Lead Configurations}

Four electrodes were defined in our 3-D model of the thorax anatomy: a point electrode in the apex of the RV (tip), a point electrode close to the previous electrode (ring), an elongated electrode running parallel to the ventricular septum (distal coil), and a large parallelepiped electrode placed in a left pectoral location (can). Electrode dimensions were chosen similar to those of commercially available devices. Distal coil length was set to $39 \mathrm{~mm}$, can width, depth, and height to $66.6 \mathrm{~mm} \times 22.7 \mathrm{~mm} \times 46.5 \mathrm{~mm}$, and the distances between the tip and the ring electrodes and between the tip and the distal coil were set to $8 \mathrm{~mm}$.

Four transvenous lead configurations were defined, namely two unipolar leads and two bipolar leads. The first unipolar configuration consisted of the tip and the can, and the second of the distal coil and the can. The true bipolar configuration was defined from the tip to the ring, and the integrated bipolar configuration was defined from the tip to the distal coil.

The sensitivity distributions of each lead configuration were obtained by using the principle of reciprocity [17], which states that the current field induced in a volume conductor when a lead is reciprocally energized with one unit of current, corresponds to the sensitivity distribution of that lead. In order to calculate the current field, we used a finite difference method (FDM) approach in the 3-D model of the human thorax. FDM allows the calculation of electric potentials and currents by dividing the anatomy into a rectangular grid forming a resistive network [26]. Using the values of tissues resistivities and the dimensions of the rectangular grid, the resistances of the network were obtained. The resistive network was then described electrically as a set of linear equations representing the potentials and currents in each node, which was solved for every lead by following iterative methods described elsewhere [26], [27].

\section{Cardiac Electrical Activity}

We implemented the state machine model for the cardiac electrical activity developed in [28]. When used to simulate cardiac electrical activity, this model has been shown to reproduce the electric restitution of both action potential duration (APD) and conduction velocity (CV), as well as curvature effects and complex dynamics, such as rotors and fibrillatory conduction. Because of its algorithmic formulation, the model has a low 
computational burden while showing a high descriptive power from a macroscopic viewpoint.

Cardiac tissue was modeled as a grid of discrete nodes. Each cardiac node was characterized by three discrete states of physiological significance, namely rest, refractory 1 , and refractory 2 , and by the transitions among them. The rules of transition among the states of each node are briefly described as follows. The transition from rest to refractoryl corresponded to the depolarization of a node, and it was formulated as a probabilistic event that depended on the amount of excitation in the neighborhood of the node and on its excitability. Excitability, in turn, was quantified using the $\mathrm{CV}$ restitution curve, according to which the longer one node is at rest, the more excitable it becomes and the more likely it is that it changes to refractoryl for a given amount of excitation in its vicinity. The transition from refractoryl to rest through refractory2, that corresponds to the repolarization of a node, depended on the current value of the APD in the node, which was estimated from the APD restitution curve.

Each ventricular node was assigned a membrane voltage based on a template action potential that is scaled in time to the current value of the APD and to the node's state. The time-varying current source distribution at node $v, \mathbf{J}(v, t)$ was solved from the voltage differences and conductivities between neighboring nodes. In this manner, by simulating cardiac activation, we simulated the generation of a distribution of bioelectric dipole sources by cardiac tissue.

\section{Computer Simulation Conditions}

We concentrated on the simple case of when the VM is stimulated by a train of ectopic beats. In order to include the variability in the estimation of the ResV due to the simulated dynamics of the heart, four stimulation foci were considered in the simulations. They were located in the ventricular apex, in the septum, in the RV free wall and in the LV free wall. The stimulation rate was set to $1 \mathrm{~Hz}$ and the simulation time was $20 \mathrm{~s}$. Simulated EGM were sampled at 1000 samples per second. For the estimation of the ResV, we considered the cases when $\alpha=0.05$ and $\alpha=0.1$.

\section{RESULTS}

\section{A. Isosensitivity Surfaces}

Fig. 4 shows the magnitude of the sensitivity distribution for each ICD lead configuration normalized to the maximum magnitude, in a cross section of the VM. As expected, the magnitude of the sensitivity is larger in the vicinity of the location of the electrodes and subsequently, it decreases with the distance. Additionally, true bipolar configurations present the largest maximum sensitivity in the VM, followed by unipolar configurations, with a maximum sensitivity of approximately half of the true bipolar case, and finally, integrated bipolar configurations with a maximum sensitivity of nearly a quarter of it. Isosensitivity maps highlight regional differences in the distribution of the sensitivity between leads. For true bipolar configurations, the sensitivity is largest at the apex of the RV, where the tip electrode is located, and decreases very rapidly radially. It can be

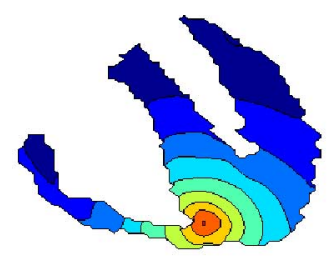

(a)

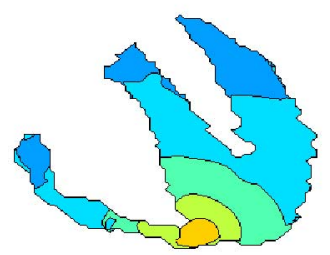

(c)

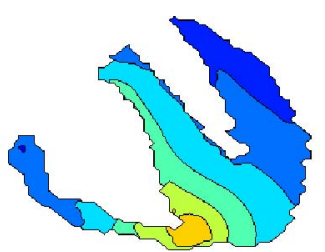

(b)

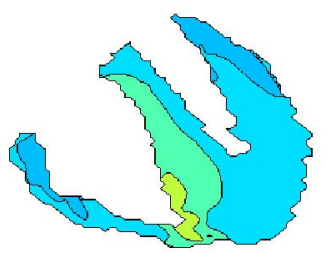

(d)

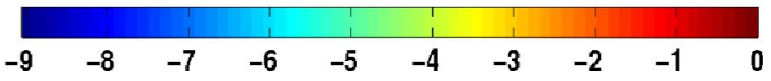

Fig. 4. Logarithm of the magnitude of the sensitivity distribution normalized to the maximum magnitude in a cross section of the VM for (a) true bipolar, (b) integrated bipolar, (c) tip-can unipolar, and (d) coil-can unipolar configurations.

observed that the magnitude of the sensitivity decreases as much as 9 orders of magnitude within the VM. Integrated bipolar configurations on the other hand, are characterized by a sensitivity distribution that is largest along the septum, consistent with the location of the coil electrode. Although less than in the case of true bipolar configurations, the gradient of the magnitude of the sensitivity is steep, resulting in a maximum difference of 4 orders of magnitude between different regions, and its direction is perpendicular to the septum. Unipolar configurations are characterized by lower sensitivities that are more uniform throughout the ventricles. In both cases, tip-can and coil-can, the magnitude of the sensitivity distribution differs as much as 2 orders of magnitude. Nevertheless, whereas the sensitivity of tip-can unipolar configurations decreases radially from the apex of the RV, it decreases perpendicularly to the septum in the case of the coil-can unipolar configurations.

\section{B. Lead Resolution Volume}

In Fig. 5, we represent the normalized MSD, $\operatorname{MSD}_{n}\left\{V_{O}\right\}$, as a function of the size of the ventricular region $V_{O}$ where it is evaluated. Error bars quantify differences due to the selection of the origin of the stimulation sequence in our simulations. As it would be expected, for every configuration the normalized MSD decreases for ventricular regions of increasing size. When the size of the ventricular region $V_{O}$ is infinitesimally small $\operatorname{MSD}_{n}\left\{V_{O}\right\}=1$, whereas for a ventricular region that comprises the whole $\mathrm{VM} \mathrm{MSD}_{n}\left\{V_{O}\right\}=0$. By comparing panels (a)-(d), we can observe that the rate of decrease of $\operatorname{MSD}_{n}\left\{V_{O}\right\}$ with the size of $V_{O}$ is different for each configuration. True bipolar configurations reveal the steepest initial decrease, while coil-can unipolar lead configurations have the slowest decrease. Integrated bipolar configurations and tip-can 


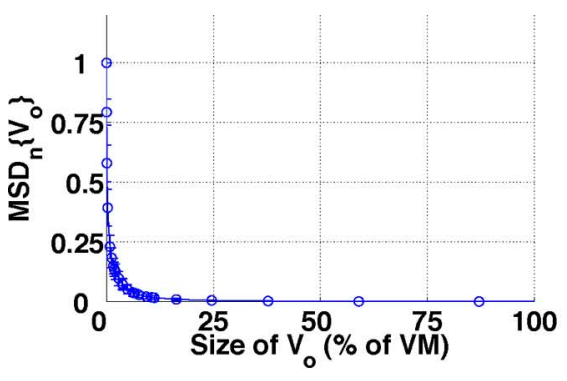

(a)

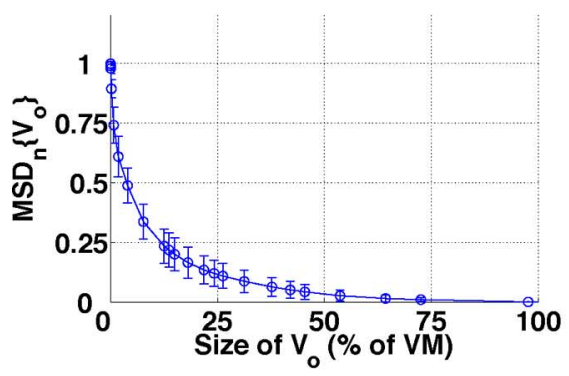

(c)

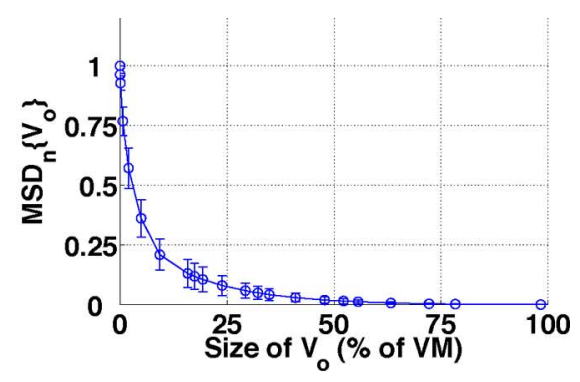

(b)

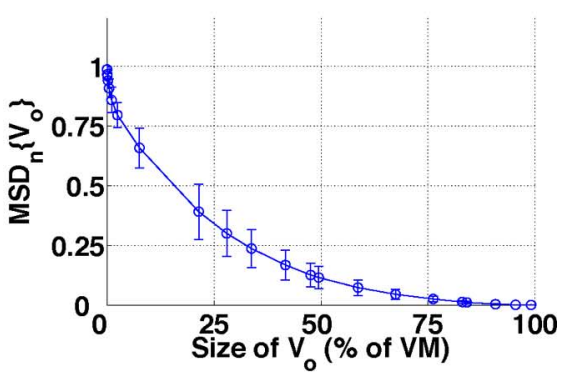

(d)

Fig. 5. Dependence of $\operatorname{MSD}_{n}\left(V_{O}\right)$ on the size of the ventricular region $V_{O}$ for (a) true bipolar, (b) integrated bipolar, (c) tip-can unipolar, and (d) coil-can unipolar configurations. Error bars correspond to mean \pm standard deviation.

TABLE I

LEAD RESOLUTION VOLUME

\begin{tabular}{|c|c|c|}
\hline Lead configuration & Resolution $(\alpha=0.05)$ & Resolution $(\alpha=0.1)$ \\
\hline True bipolar & $7 \%$ & $3 \%$ \\
Integrated bipolar & $35 \%$ & $20 \%$ \\
Tip-can unipolar & $45 \%$ & $30 \%$ \\
Coil-can unipolar & $70 \%$ & $50 \%$ \\
\hline
\end{tabular}

unipolar configurations have an intermediate behavior, as a consequence of the rate at which the magnitude of the sensitivity decreases with the distance which as revealed, is steepest for true bipolar configurations and slowest for unipolar coil-can configurations.

Based on the curves relating the normalized MSD, $\mathrm{MSD}_{n}\left\{V_{O}\right\}$, and the size of the ventricular region $V_{O}$ where it is evaluated, we obtained for each configuration the mean size of the ResV as a function of $\alpha$. The results for $\alpha=0.05$ and $\alpha=0.1$ are summarized in Table I. For the same $\alpha$, it is apparent that true bipolar configurations have a narrower ResV than integrated bipolar configurations and both unipolar configurations. Unipolar tip-can configurations and integrated bipolar configurations behave in the same manner, and coil-can unipolar configurations have the widest ResV.

The distribution of the ResV throughout the VM when $\alpha=$ 0.5 is shown in Fig. 6(a)-(d) for each lead configuration. As is expected, the ResV is always located in the vicinity of the electrodes where the sensitivity is larger, which is in agreement with the analysis of the sensitivity distribution. For true bipolar configurations, the spatial resolution is located in the apex of the RV. For integrated bipolar configurations, the ResV runs along the septum following the direction of the coil. For tip-can unipolar configurations, the spatial resolution locates around the

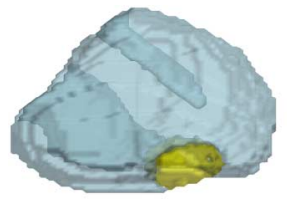

(a)

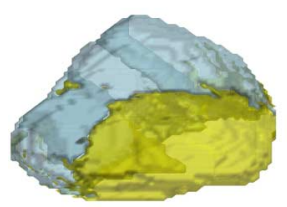

(c)

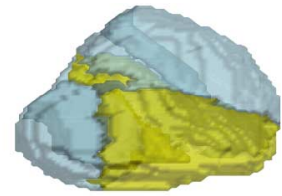

(b)

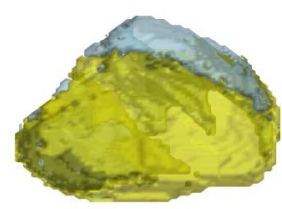

(d)
Fig. 6. Spatial resolution in the VM for (a) true bipolar, (b) integrated bipolar, (c) tip-can unipolar, and (d) coil-can unipolar configurations $(\alpha=0.05)$.

tip electrode, as in the case of the true bipolar electrode, but in this case, it has a larger extension. Finally, coil-can unipolar configurations present a much larger and more global extension than the other configurations.

\section{DISCUSSION}

Despite the importance of the notions of sensitivity and spatial resolution in analyzing EGM features and ICD performance, little attention has been paid to their quantification in ICD transvenous leads. Theoretically, since electrodes forming unipolar leads in ICD are more distant from one another than electrodes forming bipolar leads, it is generally assumed that unipolar leads sense globally, while bipolar leads sense locally. As a consequence, it is common to refer to unipolar EGM as far-field EGM and bipolar EGM as near-field EGM, although 
using both terms is misleading in this context since it does not reflect the quasi-statical nature of bioelectric phenomena [29]. Clinically it has been reported that sensing electrical events by transvenous bipolar leads depends on the availability of 1 or $2 \mathrm{~cm}^{2}$ of myocardium under the distalmost electrode [15], but to our best knowledge no quantification of the sensitivity and the spatial resolution of ICD transvenous leads has been attempted so far, either theoretically or experimentally.

The aim of this study was to quantify in the VM the sensitivity distribution and the spatial resolution of ICD transvenous leads. We formed our approach from the computation of the sensitivity distribution in a detailed 3-D model of the human thorax, and from a novel method to characterize the spatial resolution based on the concept of the ResV. By using a numerical method based on computer simulations, we were able to quantify in our model the size of the ResV of two unipolar and two bipolar ICD lead configurations and we identified their anatomical distribution throughout the VM. Our analysis revealed fundamental differences between the sensitivity distribution and the ResV of each ICD lead configuration. The quantification of the ResV could help to explain the effects of lead configuration on EGM features, and furthermore, it could contribute to improving and expanding current ICD functionality. For instance, in order to detect early some arrhythmic episodes in Brugada patients and to detect and monitor myocardial ischemia, it would be crucial to estimate the spatial resolution of current ICD transvenous leads. Additionally, leads that have a desired spatial resolution could be designed for specific applications. It is worth noting that by simply changing the origin of the activation, we obtained different estimations for the ResV, as represented by the error bars in Fig. 5. The fact that fairly similar dynamics yield different estimations of the ResV serves as a basis to state that in general, the spatial resolution is not a property of the lead system alone but of the lead system in combination with cardiac dynamics. Therefore, according to our study, spatial resolution should be interpreted as a magnitude of a statistical nature that accounts for different cardiac dynamics and can be characterized by an average behavior.

The bioelectric model that we have implemented for the computation of ICD leads sensitivity and spatial resolution has several limitations that could affect the accuracy of our results, and therefore, we should be careful when extrapolating our results to more general scenarios. First, the VHM dataset was obtained from a deceased male subject, and therefore, it does not provide a description of a human thorax in a living physiological condition. Another limitation is that we used an FDM approach for computing the lead sensitivity distributions and although FDM is easy to implement, it defines step-type boundaries that could be less exact for irregular and complex geometries [26]. On the other hand, previous studies on the application of FDM in bioelectric problems have reported that they achieve satisfactory solutions [30]-[32]. Second, the anatomical model of the VM that we used in our study was isotropic. The effects of anisotropy due to cardiac fiber orientation on the sensitivity distribution have not been previously investigated. However, in a study of defibrillation efficacy in ICD, myocardial anisotropy was found to have a small influence on the computed myocar- dial voltage gradient following defibrillation [33]. Therefore, by virtue of the principle of reciprocity, it would be expected that myocardial anisotropy did not affect significantly the sensitivity distributions of ICD leads. Third, cardiac motion could induce changes in the ResV. It has been shown that cardiac motion affects electrocardiographic potentials [34]. Since cardiac motion changes the geometry of the VM, lead sensitivity distribution could be equally affected by the motion of the heart, although its importance should be further investigated. Finally, the model of cardiac electrical activation does not include explicitly subcellular phenomena. More detailed models that include a description of cardiac electrical activity based on the bidomain paradigm [35] and human ventricular cell dynamics [36] could be developed, at the expense of an increase in computational burden. Nevertheless, since EGM signals constitute a macroscopic bioelectric phenomenon, we consider that for the purpose of our study a state machine approach has enough descriptive power.

The methodology that we have developed to investigate the spatial resolution of ICD transvenous leads could be extended in different ways. First, more elaborate bioelectric models could be implemented to improve the accuracy of our results. For instance, anisotropic myocardium and cardiac motion could be included in the definition of the model. Second, the effects on the ResV of a broader range of cardiac rhythms including ventricular and supraventricular tachyarrhythmias, could be investigated. Third, other lead parameters that have been shown to affect EGM main features and ICD sensing performance, such as interelectrode distance [8], [37], [38], and electrode location [5], size [39], and geometry [40], could also be studied. Finally, other related problems could be tackled based on the ResV characterization, including the analysis of the specificity of ICD transvenous leads to ventricular versus atrial electrical activity, and the severity of the effects of physiological noise that is generated by other electrical tissues and organs.

\section{REFERENCES}

[1] P. H. Belott and D. W. Reynolds, "Permanent pacemaker and implantable cardioverter-defibrillator implantation," in Clinical Cardiac Pacing, Defibrillation, and Resynchronization Therapy, K. A. Ellenbogen, G. N. Kay, C.-P. Lau, and B. L. Wilkoff, Eds. Philadelphia, PA/New York: Saunders/Elsevier, 2007, pp. 561-651.

[2] V. DeCaprio, P. Hurzeler, and S. Furman, "A comparison of unipolar and bipolar electrograms for cardiac pacemaker sensing," Circulation, vol. 56, no. 5, pp. 750-755, Nov. 1977.

[3] V. Parsonnet, G. H. Myers, and Y. M. Kresh, "Characteristics of intracardiac electrograms II: Atrial endocardial electrograms," Pacing Clin. Electrophysiol., vol. 3, no. 4, pp. 406-417, Jul. 1980.

[4] J. J. Langberg, W. J. Gibb, D. M. Auslander, and J. C. Griffin, "Identification of ventricular tachycardia with use of the morphology of the endocardial electrogram," Circulation, vol. 77, no. 6, pp. 1363-1369, Jun. 1988.

[5] J. M. Jenkins, "Impact of electrode placement and configuration on performance of morphological measures of intraventricular electrograms," in Proc. IEEE Comput. Cardiol., Sep. 1992, pp. 367-370.

[6] V. Menz, D. Schwartzman, D. Drachman, J. J. Michele, and S. M. Dillon, "Recording of pacing stimulus artifacts by endovascular defibrillation lead systems: Comparison of true and integrated bipolar circuits," J. Intervent. Cardiac Electrophysiol., vol. 2, pp. 269-272, Sep. 1998.

[7] B. H. Frain, K. E. Ellison, G. F. Michaud, C. H. Koo, A. E. Buxton, and M. M. Kirk, "True bipolar defibrillator leads have increased sensing latency and threshold compared with the integrated bipolar configuration,' J. Cardiovasc. Electrophysiol., vol. 18, no. 2, pp. 192-195, Feb. 2007. 
[8] M. Cooklin, R. V. Tummala, R. W. Peters, S. R. Shorofsky, and M. R. Gold, "Comparison of bipolar and integrated sensing for redetection of ventricular fibrillation,” Amer. Heart J., vol. 138, no. 1, pt. 1, pp. 133-136, Jul. 1999.

[9] A. Natale, J. Sra, K. Axtell, M. Akhtar, K. Newby, V. Kent, M. J. Geiger, M. J. Brandon, M. M. Kearney, and A. Pacifico, "Undetected ventricular fibrillation in transvenous implantable cardioverter-defibrillators. prospective comparison of different lead system-device combinations," Circulation, vol. 93, no. 1, pp. 91-98, Jan. 1996.

[10] J. J. Goldberger, G. Horvath, D. Donovan, D. Johnson, R. Challapalli, and A. H. Kadish, "Detection of ventricular fibrillation by transvenous defibrillating leads: Integrated versus dedicated bipolar sensing," J. Cardiovasc. Electrophysiol., vol. 9, pp. 677-688, Jul. 1998.

[11] P. G. Meregalli, A. A. Wilde, and H. L. Tan, "Pathophysiological mechanisms of Brugada syndrome: Depolarization disorder, repolarization disorder, or more?" Cardiovasc. Res., vol. 67, no. 3, pp. 367-378, 2005.

[12] S. Asbach, I. Weiss, B. Wenzel, and C. Bode, "Intrathoracic far-field electrocardiogram allows continuous monitoring of ischemia after total coronary occlusion," Pacing Clin. Electrophysiol., vol. 29, no. 12, pp. 1334-1340, Dec. 2006.

[13] J. L. Williams, G. S. Mendenhall, and S. Saba, "Effect of ischemia on implantable defibrillator intracardiac shock electrograms," J. Cardiovasc. Electrophysiol., vol. 19, no. 3, pp. 282-284, Mar. 2008

[14] T. J. Bunch and J. D. Day, "The diagnostic evolution of the cardiac implantable electronic device: The implantable monitor of ischemia," $J$ Cardiovasc. Electrophysiol., vol. 19, no. 3, pp. 275-81, Mar. 2008

[15] C. D. Swerdlow, J. M. Gillberg, and W. H. Olson, "Sensing and detection," in Clinical Cardiac Pacing, Defibrillation, and Resynchronization Therapy, K. A. Ellenbogen, G. N. Kay, C.-P. Lau, and B. L. Wilkoff, Eds. Philadelphia, PA/New York: Saunders/Elsevier, 2007, pp. 75160.

[16] R. Gradaus, G. Breithardt, and D. Bocker, "ICD leads: Design and chronic dysfunctions," Pacing Clin. Electrophysiol., vol. 26, no. 2, pt. 1, pp. 649657, Feb. 2003

[17] J. Malmivuo and R. Plonsey, Bioelectromagnetism: Principles and Applications of Bioelectric and Biomagnetic Fields. New York: Oxford Univ. Press, 1995

[18] S. Rush and D. A. Driscoll, "EEG electrode sensitivity-An application of reciprocity," IEEE Trans. Biomed. Eng., vol. 16, no. 1, pp. 15-22, Jan. 1969.

[19] R. C. Arzbaecher, J. M. Jenkins, S. Collins, and E. Berbari, “Atrial electrical activity: The view from the esophagus," in IEEE/EMBS Frontiers Eng. Health Care, 1979, pp. 314-318.

[20] J. Malmivuo, V. Suihko, and H. Eskola, "Sensitivity distributions of EEG and MEG measurements," IEEE Trans. Biomed. Eng., vol. 44, no. 3 , pp. 196-208, Mar. 1997

[21] J. Väisänen, O. Väisänen, J. Malmivuo, and J. Hyttinen, "New method for analysing sensitivity distributions of electroencephalography measurements," Med. Biol. Eng. Comput., vol. 46, pp. 101-108, 2008.

[22] J. Requena-Carrión, J. Väisänen, J. L. Rojo-Álvarez, J. Hyttinen, F. Alonso-Atienza, and J. Malmivuo, "Numerical analysis of the resolution of surface electrocardiographic lead systems," in Proc. FIMH, 2007, pp. 310-319.

[23] M. J. Ackerman, "The visible human project," J. Biocommun., vol. 18 p. 14, 1991.

[24] P. Kauppinen, J. Hyttinen, T. Heinonen, and J. Malmivuo, "Detailed model of the thorax as a volume conductor based on the visible human man data," J. Med. Eng. Technol., vol. 22, no. 3, pp. 126-133, May/Jun. 1998.

[25] P. Kauppinen, J. Hyttinen, P. Laarne, and J. Malmivuo, "A software implementation for detailed volume conductor modelling in electrophysiology using finite difference method," Comput. Methods Prog. Biomed., vol. 58, no. 2, pp. 191-203, Feb. 1999

[26] C. R. Johnson, "Computational and numerical methods for bioelectric field problems," Crit. Rev. Biomed. Eng., vol. 25, pp. 1-81, 1997.

[27] F. B. Sachse, Computational Cardiology: Modeling of Anatomy, Electrophysiology, and Mechanics. Berlin, Germany: Springer-Verlag, 2004.

[28] F. Alonso-Atienza, J. Requena-Carrión, A. García-Alberola, J. L. Rojo-Álvarez, J. J. Sánchez-Muñoz, J. Martinez-Sánchez, and M. ValdésChávarri, "A probabilistic model of cardiac electrical activity based on a cellular automata system," Revista Española Cardiol., vol. 58, no. 1, pp. 41-47, Jan. 2005.

[29] R. Plonsey and D. B. Heppner, "Considerations of quasi-stationarity in electrophysiological systems," Bull. Math. Biophys., vol. 29, no. 4, pp. 657-664, Dec. 1967.
[30] L. Lemieux, A. McBride, and J. W. Hand, "Calculation of electrical potentials on the surface of a realistic head model by finite differences," Phys. Med. Biol., vol. 41, no. 7, pp. 1079-1091, 1996.

[31] B. Vanrumste, G. V. Hoey, R. V. de Walle, M. R. D’Have, I. A. Lemahieu, and P. A. Boon, "The validation of the finite difference method and reciprocity for solving the inverse problem in EEG dipole source analysis," Brain Topogr., vol. 14, no. 2, pp. 83-92, 2001.

[32] L. Jing, S. Zhu, and B. He, "A finite difference method for solving the three-dimensional EEG forward problem," in Proc. 27th Annu. Int. Conf. Eng. Med. Biol. Soc., Jan., 2005, pp. 1540-1543.

[33] Y. Wang, D. Haynor, and Y. Kim, "An investigation of the importance of myocardial anisotropy in finite-element modeling of the heart: methodology and application to the estimation of defibrillation efficacy," IEEE Trans. Biomed. Eng., vol. 48, no. 12, pp. 1377-1389, Dec. 2001.

[34] Q. Wei, F. Liu, B. Appleton, L. Xia, N. Liu, S. Wilson, R. Riley, W. Strugnel, R. Slaughter, R. Denman, and S. Crozier, "Effect of cardiac motion on body surface electrocardiographic potentials: an MRI-based simulation study," Phys. Med. Biol., vol. 51, no. 14, pp. 3405-3418, 2006.

[35] C. S. Henriquez, "Simulating the electrical behavior of cardiac tissue using the bidomain model," Crit. Rev. Biomed. Eng., vol. 21, no. 1, pp. 1-77, 1993

[36] K. H. W. J. ten Tusscher, D. Noble, P. J. Noble, and A. V. Panfilov, "A model for human ventricular tissue," Amer. J. Physiol. Heart Circ. Physiol., vol. 286, no. 4, pp. H1573-1589, 2004.

[37] D. L. Derfus and T. C. Pilkington, "Assessing the effect of uncertainty in intracavitary electrode position on endocardial potential estimates," IEEE Trans. Biomed. Eng., vol. 39, no. 7, pp. 676-681, Jul. 1992.

[38] X. Min, S. Meyer, and R. Mehra, "Computer modeling and experimental validation on effect of bipolar electrode spacing on near field and far field electrogram amplitudes," in Proc. IEEE Comput. Cardiol., 1999, pp. 201-204.

[39] G. H. Myers, Y. M. Kresh, and V. Parsonnet, "Characteristics of intracardiac electrograms," Pacing Clin. Electrophysiol., vol. 1, no. 1, pp. 90-103, Jan. 1978.

[40] W. Sun and X. Min, "An integral equation model for intracardiac electrogram sensing," IEEE Trans. Biomed. Eng., vol. 44, no. 12, pp. 1237-1242, Dec. 1997

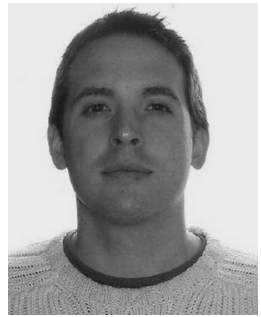

Jesús Requena-Carrión (M'08) received the Electrical Engineering and Ph.D. degrees from the Universidad Carlos III de Madrid, Madrid, Spain.

Since 2006, he has been with the Department of Signal Theory and Communications, Universidad Rey Juan Carlos, Madrid, where he is currently an Assistant Lecturer. His current research interests include the area of signal processing and computer modeling in cardiac electrophysiology.

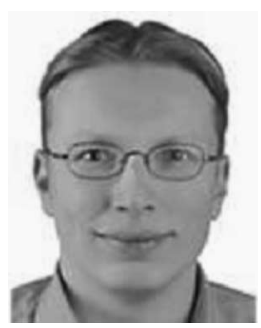

Juho Väisänen received the M.Sc. degree in biomedical engineering in 2003 from Tampere University of Technology (TUT), Tampere, Finland, where he is currently working toward the $\mathrm{Ph}$. D. degree in biomedical engineering.

His current research interests include modeling of cardiac and neural measurements. 


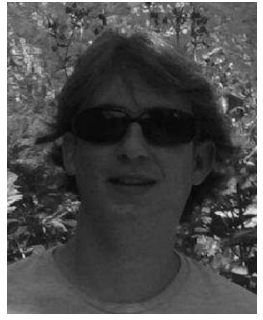

Felipe Alonso-Atienza (S'03-M'08) received the M.Sc. degree in telecommunication engineering and the Ph.D. degree from the Universidad Carlos III de Madrid, Madrid, Spain, in 2003 and 2008, respectively.

$\mathrm{He}$ is currently an Associate Professor at the Department of Signal Theory and Communications, Universidad Rey Juan Carlos, Madrid. His current research interests include cardiac modeling, bioelectromagnetism, and applications of statistical learning to signal processing with particular attention to car-

diac signals analysis.

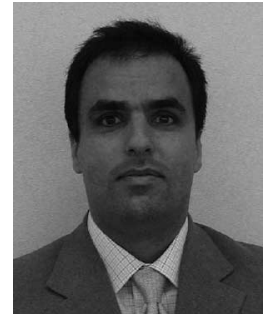

Francisco Javier Ramos-López (M'95) received the Telecommun. Eng. and Ph.D. degrees in telecommunications from the Universidad Politécnica de Madrid, Spain, in 1990 and 1995, respectively.

Between 1992 and 1995, he was involved in several research projects at Purdue University, West Lafayette, IN, where he was engaged in the field of signal processing for communications. During 1996, he was a Post-Doctoral Research Associate at Purdue University. From 1997 to 2003, he was an Associate Professor at Universidad Carlos III de Madrid, Madrid. Since 2003, he has been the Dean of the Telecommunications Engineering School, Universidad Rey Juan Carlos, Madrid. His current research interests include the areas of signal processing, mobile communications, and networking.

Dr. Ramos received the COIT-Ericsson Award for the best Ph.D. dissertation on mobile communications in 1996.

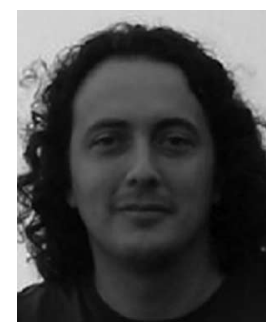

José Luis Rojo-Álvarez (M’01) received the Telecommun. Eng. degree from the University of Vigo, Vigo, Spain, in 1996, and the Ph.D. degree in telecommunications from the Polytechnical University of Madrid, Madrid, Spain, in 2000.

Since 2006, he has been an Associate Professor at the Department of Signal Theory and Communications, University Rey Juan Carlos, Madrid. He is the coauthor of more than 40 papers and more than 100 international conference communications. His current research interests include statistical learning theory, digital signal processing, and complex system modeling, with applications to digital communications and to cardiac signal and image processing. sis, arrhythmia mechanisms, and cardiac signal processing.
Arcadi García-Alberola received the M.D. and de Valencia, Valencia, Spain, in 1982 and 1991, respectively.

Professor of medicine at the Hospital Universitario Murcia, Spain, where he is currently the Chief of the Laboratory of Cardiac Electrophysiology. He has authored or coauthored more than 120 scientific papers and more than 50 communications in cardiac 